\title{
Estrutura e composição da comunidade de Trichoptera (Insecta) de rios e áreas alagadas da bacia do rio Suiá-Miçú, Mato Grosso, Brasil
}

\author{
Denis Silva Nogueira ${ }^{1}$, Helena Soares Ramos Cabette $^{2} \&$ Leandro Juen ${ }^{3}$
}

1. Programa de Pós-Graduação em Ecologia e Evolução, Universidade Federal de Goiás, 74001-970, Goiânia, Goiás, Brasil. (dnogueira_bio@yahoo.com.br)
2. Programa de Pós-Graduação em Ecologia e Conservação, Universidade do Estado de Mato Grosso, 78690-000, Nova Xavantina, Mato Grosso, Brasil. (hcabette@uol.com.br)
3. Instituto de Ciências Biológicas, Universidade Federal do Pará, Rua Augusto Correia, 01 Bairro Guamá, 66075-110, Belém, Pará, Brasil. (leandrojuen@yahoo.com.br)

\begin{abstract}
Structure and composition of Trichoptera (Insecta) community in streams and wetlands of Suiá-Miçú River Basin, Mato Grosso, Brazil. The composition, abundance and species diversity of Trichoptera immatures was studied in 12 tributaries of Suiá-Miçú River Basin, a mosaic of wetlands, streams and rivers tributary of the Xingu River in the Cerrado-Amazonian Forest transition zone in Mato Grosso, Brazil. The varying sizes, flow types and conservation levels of the tributaries were sampled through three periods between 2007 and 2008 by the use of fixed transects along the environments margins. A total of 867 larvae was collected (divided in seven families, 17 genera, 49 species/ morphospecies) with the most abundant and rich families being Hydropsychidae and Leptoceridae and the most abundant species Leptonema $\operatorname{sparsum}(\mathrm{n}=370)$. There has been loss of species richness in impacted, large and lentic environments. Composition variations related to the flow, the vegetation type and the interaction between conservation levels and flow was detected. Conservation levels, width and the quantitative habitat integrity index (HII) did not influence the Trichoptera composition. These results may encourage further investigation of impact effects on aquatic insect compositions and pattern of distribution in the transition area between the Cerrado and the Amazonian Forest.
\end{abstract}

KEYWORDS. Diversity, larvae, stream ecology, biomonitoring, Xingu River.

RESUMO. A composição, abundância e riqueza de Trichoptera imaturos foram estudadas em 12 tributários da bacia do rio Suiá-Miçú, afluente do rio Xingu, um mosaico de áreas alagadas, córregos e rios na área de transição Cerrado - Floresta Amazônica na região leste de Mato Grosso, Brasil. Larvas de insetos aquáticos foram amostradas em transectos nas margens de tributários lênticos e lóticos com tamanho e níveis de conservação variáveis, em três períodos entre 2007 e 2008. Foram coligidas 867 larvas (sete famílias, 17 gêneros, 45 morfoespécies), sendo Hydropsychidae e Leptoceridae as famílias mais abundantes e ricas, e Leptonema sparsum a espécie mais abundante $(\mathrm{n}=370)$. Houve perda de riqueza de espécies em ambientes impactados e naqueles de maior porte ou de fluxo lêntico. Foi possível detectar variações na composição relacionadas ao fluxo, tipo de vegetação e na interação entre o nível de conservação e fluxo. O nível de conservação, a largura e o índice quantitativo de integridade de hábitat (IIH) não influenciaram a composição de Trichoptera isoladamente. Estes resultados podem fomentar novas investigações dos efeitos dos impactos sobre a composição da entomofauna aquática na transição entre o Cerrado e a Floresta Amazônica.

PALAVRAS-CHAVE. Diversidade, larvas, ecologia de riachos, biomonitoramento, rio Xingu.

Insetos aquáticos são organismos extremamente vulneráreis a alterações ambientais e constituem um importante componente da diversidade e integridade de ambientes aquáticos (Merritt \& Cummins, 1984; Rosenberg \& Resh, 1993). O conhecimento da composição faunística e da estrutura das comunidades de insetos é vital para o monitoramento e detecção de mudanças na qualidade da água, permitindo a mensuração da integridade ecológica de rios e córregos (Rosenberg \& Resh, 1993; Bonada et al., 2006).

A ordem Trichoptera compreende um dos grupos mais diversos entre os insetos aquáticos, com cerca de 13000 espécies distribuídas no mundo (Holzenthal et al., 2007). Apesar da grande diversidade de espécies conhecidas para o Brasil, com aproximadamente 550 registros (SANTOS et al., 2011), somente oito eram anotadas para o estado do Mato Grosso até 2003 (PAPROCKI et al., 2004). Levantamentos preliminares têm revelado que a diversidade de espécies no estado ainda é extremamente subestimada, e a adição de novos registros tem sido provida principalmente na região leste, onde os estudos sobre a fauna tem se intensificado (Nogueira \& CABETte, 2011).

Os tricópteros se destacam no monitoramento da qualidade da água por apresentarem elevada riqueza e abundância, níveis variados de sensibilidade a alterações físicas e químicas e à poluição dos ecossistemas aquáticos
(Rosenberg \& Resh, 1993; Wiggins, 1996; Angrisano \& KOROB, 2001). As larvas são elos na transferência de energia entre os diferentes níveis das teias tróficas (Merritt \& Cummins, 1984) e, muitas vezes, constituem grande proporção da biomassa de insetos em riachos (Flint et al., 1999). Apresentam grande diversidade de comportamentos para aquisição de alimento, sendo representada em todas as categorias funcionais dos macroinvertebrados (Cummins \& KLUG, 1979; Cummins \& Merritt, 2008). Esta diversidade funcional tem sido atribuída à produção de seda por suas glândulas labiais (MACKAY \& WigGins, 1979), o que as possibilitou a ocupação de vários habitats de ecossistemas aquáticos lênticos e lóticos (Fuint et al., 1999).

Ecossistemas aquáticos tropicais têm sido submetidos a intensos impactos, sendo a agricultura e a pecuária os principais responsáveis pelas modificações na vegetação ripária (TownSEND et al., 1997; WANTZEN et al., 2006). No estado de Mato Grosso as estimativas da redução da cobertura de vegetação original relacionada às atividades humanas foram de $785 \mathrm{a} 2.150 \mathrm{~km}^{2} /$ ano somente entre o período de 2001 a 2004 (MORTON et al., 2006). Essas atividades têm levado a danos consideráveis sobre as áreas de reserva legal e Áreas de Preservação Permanente (APPs), com taxas elevadas de conversão da vegetação natural em pastagens e monoculturas (Pongratz et al., 2006). 
A despeito de sua importância para a dinâmica energética dos ambientes onde vivem, levantamentos da entomofauna aquática são escassos na região leste de Mato Grosso (Juen et al., 2007; Dias-Silva et al., 2010; Shimano et al., 2010; Nogueira \& Cabette, 2011). Poucos estudos têm avaliado os efeitos dos impactos antrópicos sobre a composição e estrutura das comunidades de Trichoptera (Oliveira \& Froehlich, 1997; Oliveira \& Bispo, 2001; Bispo et al., 2006), e um número ainda mais reduzido têm investigado a fauna de áreas alagadas (МАLтснік et al., 2009). Especialmente na região da transição Cerrado-Amazônia, os estudos ecológicos são inexistentes, sendo os poucos registros pontuais (Nogueira \& CABETTE, 2011). Diante desse cenário, o objetivo deste trabalho foi inventariar e descrever os padrões de distribuição espacial, estrutura e composição das comunidades de tricópteros imaturos em rios e córregos da bacia do rio Suiá-Miçú, Mato Grosso.

\section{MATERIAL E MÉTODOS}

O rio Suiá-Miçú está situado entre os paralelos $11^{\circ} 15^{\prime}$ a $13^{\circ} 40^{\prime} \mathrm{S}$ e $53^{\circ} 15^{\prime}$ a $51^{\circ} 15^{\prime} \mathrm{W}$, na transição entre o Cerrado e Floresta Amazônica (ver figura 1 em SHIMANo et al., 2010). Constitui um dos principais afluentes da margem direita do rio Xingu, drenando áreas de planície entre os municípios de Água Boa, Canarana, Ribeirão Cascalheira e Querência, a nordeste do estado de Mato Grosso. O clima da região é do tipo tropical sazonal com uma estação seca (de maio a outubro) e uma chuvosa (de novembro a abril). Seguindo a classificação de KöPPEN (1931), o clima predominante na região é do tipo Savana (Aw) principalmente ao sul da bacia, onde predomina a fitofisionomia de cerrado senso stricto; mas há microrregiões de subtipo Monções (Am) e Tropical Chuvoso (A) mais ao norte, onde as formações florestais de cerradão de Hirtella glandulosa Spreng. e mata seca são mais comuns (sensu RATTER et al., 1978). A precipitação anual média é de $1370 \mathrm{~mm}$ e as temperaturas máximas e mínimas variam em torno de $32,7^{\circ} \mathrm{C}$ e $17,0^{\circ} \mathrm{C}$, respectivamente (RATTER et al., 1978).

O estudo foi conduzido em rios e córregos ao longo da bacia do rio Suiá-Miçú e em um de seus principais tributários, o rio Darro, sendo que os ambientes variam de $1^{\mathrm{a}}$ à $6^{\mathrm{a}}$ ordem segundo a classificação de STRAHLER (1957). As coletas foram realizadas em três períodos: setembro (seca), dezembro (chuva) de 2007 e em maio (vazante) de 2008. Foram amostrados 12 ambientes que variaram amplamente em tamanho, velocidade do fluxo de água, tipo de vegetação ripária e conservação. Nos ambientes lóticos os substratos amostrados consistiram de raízes de barranco e vegetação submersa, folhas em poções e retidas em corredeiras, gravetos e pedaços de troncos, pedras, cascalho e areia. Nos ambientes brejosos os substratos consistiram principalmente de macrófitas submersas, raízes e matéria em decomposição misturada à areia. As amostras foram obtidas em transecções fixas de $100 \mathrm{~m}$, divididas em 20 segmentos de cinco metros, sendo as amostras obtidas através de um coador de $18 \mathrm{~cm}$ de diâmetro e malha de $0,05 \mathrm{~mm}$, replicando-se três vezes uma porção do substrato presente ao longo da margem de cada segmento (modificado de Ferreira-PeruQuetti \& De Marco, 2002; Shimano et al., 2010; Dias-Silva et al., 2010). O material foi separado em campo e conservado em álcool etílico a 85\%. A identificação dos gêneros se deu com chaves dicotômicas (ANGRISANO \& Kоrob, 2001; Pes et al., 2005).

Em Trichoptera a identificação específica das larvas é feita somente através de criação para a obtenção de adultos ou por métodos de associação de larvas, pupas e adultos farados (Milne, 1938; Flint, 1974). No presente estudo, as larvas foram morfoespeciadas com base em caracteres morfológicos padrão nas descrições de imaturos (Holzenthal, 1988; 1995; Holzenthal \& Pes, 2004; Pes et al., 2008). A variação entre instares foi desconsiderada para o estabelecimento das morfoespécies e larvas muito jovens retiradas das análises. Os exemplares foram depositados na Coleção Zoobotânica "James Alexander Ratter" (CZNX), na Universidade do Estado de Mato Grosso, Nova Xavantina, MT, Brasil.

A definição do nível de conservação dos ambientes foi estabelecida a partir do Indice de Integridade de Hábitat (IIH). O IIH é composto por 12 questões que avaliam a largura da vegetação ripária e seu estado de conservação dentro de uma faixa de $50 \mathrm{~m}$, a estrutura dos barrancos, a heterogeneidade em um trecho de 100 $\mathrm{m}$ ao longo dos ambientes, tipo de substratos, dentro outros fatores (Nessimian et al., 2008). O índice varia de zero a um, indicando um gradiente crescente de integridade. Neste estudo, o IIH oscilou de 0,39 a 0,74 , e foi utilizado para classificar os ambientes em três níveis de conservação: impactado $(0,39$ a 0,60$)$, alterado $(0,61$ a 0,68$)$ e conservado (de 0,69 a 0,74$)$. O tamanho dos ambientes variou de poucos metros a mais de $100 \mathrm{~m}$ de largura, sendo independente da ordem de magnitude $\left(\mathrm{F}_{(5,11)}=0,61, \mathrm{R}^{2}=0,34, \mathrm{p}=0,84\right)$. Quanto à vegetação, os locais foram classificados em brejo (varjão com buritizais e macrófitas), pasto (principalmente nos locais degradados), cerrado e mata.

A riqueza local de espécies foi estimada utilizando o Jackknife de primeira ordem. Este procedimento reduz o efeito das espécies dominantes e dá maior peso às espécies raras, o que permite comparações mesmo quando as amostras são pequenas (HELTSHE \& ForRESTER, 1983; Brose et al., 2003; Hortal et al., 2006). As estimativas da riqueza foram realizadas através do programa EstimateS Win 7.5.0 (COLWELL, 2005).

A composição das comunidades de Trichoptera foi analisada usando a análise de classificação divisiva - TWISPAN. A classificação é hierárquica e gera agrupamentos para grupos de sítios mais similares em composição, onde os ramos das divisões são 
representados por espécies ou conjuntos de espécies indicadoras ou preferenciais. O método separa cada espécie em categorias de pseudo-espécies de acordo com uma escala de abundância e utiliza a ocorrência destas pseudo-espécies nas parcelas para refinar os agrupamentos. Os autovalores gerados representam a contribuição dos conjuntos de espécies e amostras em cada divisão. No presente estudo, foram considerados os seguintes níveis de abundância para estabelecer as pseudo-espécies: zero, dois, cinco, 10 e 20. Assim, por exemplo, para uma dada espécie $i$ que apresente seis indivíduos na amostra $A$ receberá o nível três (entre cinco e 10 indivíduos) para esta amostra, a espécie $j$ que apresenta 25 indivíduos em uma amostra recebe o nível cinco $(>20)$. Cada espécie recebe um diferente nível de abundância para cada local onde ocorre, dependendo do número de indivíduos na comunidade local. A análise foi realizada no programa PC-Ord (McCunE \& MEFFord, 1999).

$\mathrm{O}$ efeito das características ambientais sobre a variação na composição de espécies entre os locais da bacia foi avaliado usando uma análise multivariada não paramétrica de permutação de matrizes - PER-MANOVA (ANDERSON, 2001). Foram utilizadas variáveis preditoras pré-selecionadas visando diminuir a quantidade de informação retida no resíduo. Como a largura e o IIH e suas interações com as outras variáveis não afetaram a composição faunística ( $\mathrm{p}>0,05)$, estas variáveis foram excluídas da análise que avaliou o efeito dos demais preditores devido ao baixo número de graus de liberdade, sendo apresentados somente seus efeitos individuais sobre a composição de espécies (ver Tab. II). A matriz de dissimilaridade foi obtida utilizando o método Bray-Curtis com auxílio da função vegdist, a partir de dados de abundância da comunidade logaritimizados $[\log (\mathrm{x}+1)]$. A PER-MANOVA foi realizada utilizando a função adonis, sendo os níveis de significância (razão entre as estatísticas F) obtidos com 999 aleatorizações. Estas análises foram realizadas utilizando o pacote vegan (OKsanen et al., 2010) do programa R (R DeVELopment Core Team, 2010).

\section{RESULTADOS}

Foram coligidos 867 imaturos de Trichoptera distribuídos em sete famílias, 17 gêneros e 49 morfoespécies (Tabela I). Leptoceridae com 18 e Hydropsychidae com 17 morfoespécies foram as famílias mais ricas. Hydropsychidae constituiu 76,75\% da abundância total, tendo Leptonema $(\mathrm{n}=426)$ como o gênero mais abundante da família representado por três espécies. Leptonema sparsum Ulmer, 1905 totalizou $42,67 \%$ da abundância total de larvas $(n=370)$, seguida de Macrostemum sp. 2 (9,69\%, n=84), L. maculatum Mosely, 1933 (6,34\%, n=55), Macronema sp. 4 (3,69\%, $\mathrm{n}=32)$ e Cernotina sp. $1(3,46 \%, \mathrm{n}=30)$, e compuseram as espécies de maior amplitude de distribuição na bacia; os demais táxons representaram menos de três por cento da abundância total de tricópteros.

Entre os locais analisados, o RIBET2 foi o de maior abundância, representando $42,67 \%$ dos tricópteros coletados $(\mathrm{n}=370)$, seguido do CRL com $16,83 \%(\mathrm{n}=146)$ e do CRSRI com $12,68 \%(\mathrm{n}=110)$. Os locais menos abundantes tiveram menos de 20 larvas e contribuíram juntos com apenas $5 \%$ dos exemplares coletados (Tabela I). O CRL apresentou a maior riqueza, com 21 $\pm 2,16$ morfoespécies (média \pm desvio padrão), seguido do CRSRI com $16 \pm 2,52$ e do RIBET2 com $15 \pm 3,39$, enquanto a menor riqueza foi registrada no RISU3 com apenas três (Fig. 1).

A análise classificatória divisiva (TWISPAN) revelou diferenças na composição faunística entre os locais, mostrando agrupamentos relacionados ao fluxo e conservação, tipo de vegetação e o nível de impacto (Fig. 2). Na primeira divisão $(\lambda=0,55)$ foi verificada a formação de um agrupamento positivo entre os ambientes lóticos e de cerrado senso stricto (RISU1, RISU2, RIPB) mais o RISUZ (na transição Cerrado-Amazônia), que se separaram do RISU3 na mata seca, com Macronema sp. 2 e Chimarra sp. 4 como as espécies indicadoras. Os ambientes do cerrado s.s. foram representados pela espécie preferencial L. sparsum. Na parte negativa do diagrama, ambientes abertos e alagados, impactados e o CRL (lótico preservado) compuseram um agrupamento heterogêneo, representados na primeira divisão por Macronema sp. 4, Oecetis sp. 4 e Smicridea truncata Flint, 1974. Na segunda divisão do eixo negativo do diagrama, o CRL se separou do grupo formado pelos ambientes impactados e brejosos e teve Marilia sp. 1 como espécie indicadora. A divisão subsequente isolou o RID do grupo formado pelos ambientes impactados e brejosos $(\lambda=0,55)$, tendo Amphoropsyche sp. 3 como a espécie indicadora. Os ambientes brejosos (CRBJ e CRSRI), com Cyrnellus sp.1 como espécie indicadora, formaram um agrupamento isolado de riachos impactados por pastagem (RIBET1, RIBET2 e CRTB).

Adicionalmente, a análise PER-MANOVA indicou variação significativa na dissimilaridade de composição faunística com relação aos fatores analisados (Tabela II). Houve efeito significativo do tipo de fluxo $\left(\mathrm{F}_{(1,11)}=2,81\right.$; $\left.\mathrm{R}^{2}=0,14 ; \mathrm{p}=0,02\right)$, o tipo de vegetação $\left(\mathrm{F}_{(3,11)}=1,989\right.$; $\left.\mathrm{R}^{2}=0,27 ; \mathrm{p}=0,01\right)$ e da interação entre o tipo de fluxo e o nível de conservação $\left(F_{(1,11)}=3,89 ; R^{2}=0,20 ; p=0,01\right)$ sobre a matriz de dissimilaridade. O IIH, o nível de conservação e a largura não tiveram efeito sobre a matriz de dissimilaridade quando avaliados isoladamente.

\section{DISCUSSÃO}

Em ecossistemas aquáticos os padrões de distribuição da abundância e composição são resultantes da interação entre o hábito das espécies, condições físicas dos habitats (e.g. substrato, correnteza, vegetação marginal, largura, profundidade), parâmetros daágua (e.g. 
Tab. I. Distribuição e composição da comunidade de Trichoptera da bacia do rio Suiá-Miçú, Estado do Mato Grosso, em 2007/2008 (CRSRI, córrego Sucuri; CRBJ, córrego Brejão; CRTB, córrego transição-brejo; CRL, córrego Lúcio; RISU1, rio Suiá-Miçú, ponto 1; RISU2, rio Suiá-Miçú, ponto 2; RISU3, rio Suiá-Miçú, ponto 3; RISUZ, rio Suiazinho; RID, rio Darro; RIPB, rio Piabanha; RIBET1, rio Betis, ponto 1; RIBET2, rio Betis, ponto 2).

\begin{tabular}{|c|c|c|c|c|c|c|c|c|c|c|c|c|c|}
\hline \multirow{2}{*}{ Espécies/morfoespécies } & \multicolumn{13}{|c|}{ Localidades } \\
\hline & 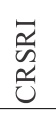 & 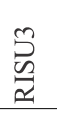 & 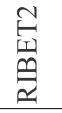 & 产 & 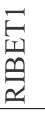 & 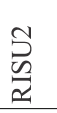 & $\overrightarrow{0}$ & 胥 & $\stackrel{5}{\mathscr{2}}$ & $\underset{\simeq}{\stackrel{\theta}{a}}$ & 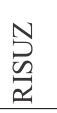 & $\overrightarrow{0}$ & 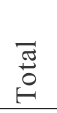 \\
\hline Amazonatolica hamadae Holz. \& Pes, 2004 & 0 & 0 & 0 & 0 & 0 & 0 & 0 & 0 & 0 & 0 & 0 & 6 & 6 \\
\hline Amphoropsyche sp. 1 & 0 & 0 & 0 & 0 & 0 & 0 & 0 & 0 & 0 & 0 & 1 & 2 & 3 \\
\hline Amphoropsyche sp. 2 & 0 & 0 & 0 & 0 & 0 & 0 & 0 & 0 & 0 & 2 & 1 & 0 & 3 \\
\hline Amphoropsyche sp. 3 & 0 & 0 & 0 & 0 & 0 & 0 & 0 & 0 & 0 & 1 & 0 & 0 & 1 \\
\hline Cernotina sp. 1 & 1 & 8 & 0 & 3 & 1 & 1 & 0 & 3 & 2 & 0 & 5 & 6 & 30 \\
\hline Chimarra sp. 1 & 0 & 0 & 0 & 3 & 0 & 5 & 0 & 0 & 6 & 0 & 0 & 0 & 14 \\
\hline Chimarra sp. 2 & 0 & 0 & 0 & 2 & 0 & 2 & 0 & 0 & 0 & 0 & 0 & 0 & 4 \\
\hline Cyrnellus sp. 1 & 2 & 0 & 0 & 1 & 0 & 0 & 2 & 0 & 2 & 0 & 0 & 0 & 7 \\
\hline Cyrnellus sp. 2 & 4 & 0 & 0 & 0 & 0 & 0 & 4 & 0 & 0 & 1 & 0 & 0 & 9 \\
\hline Cyrnellus sp. 3 & 0 & 0 & 0 & 0 & 0 & 0 & 1 & 0 & 0 & 0 & 0 & 0 & 1 \\
\hline Leptonema amazonense Flint, 1978 & 0 & 0 & 0 & 0 & 0 & 0 & 0 & 0 & 0 & 0 & 0 & 1 & 1 \\
\hline L. maculatum Mosely, 1933 & 22 & 0 & 4 & 0 & 0 & 0 & 0 & 0 & 0 & 0 & 0 & 29 & 55 \\
\hline L. sparsum Ulmer, 1905 & 45 & 0 & 251 & 8 & 0 & 7 & 17 & 0 & 12 & 0 & 5 & 25 & 370 \\
\hline Macronema sp. 3 & 12 & 0 & 1 & 0 & 0 & 0 & 2 & 4 & 0 & 0 & 0 & 0 & 19 \\
\hline Macronema sp. 4 & 3 & 0 & 2 & 0 & 0 & 0 & 2 & 6 & 1 & 18 & 0 & 0 & 32 \\
\hline Macronema sp. 5 & 1 & 0 & 0 & 2 & 1 & 9 & 0 & 0 & 3 & 0 & 1 & 0 & 18 \\
\hline Macronema sp. 6 & 2 & 0 & 0 & 0 & 0 & 0 & 0 & 3 & 0 & 0 & 0 & 7 & 12 \\
\hline Macronema sp. 7 & 1 & 0 & 0 & 1 & 0 & 0 & 0 & 0 & 0 & 5 & 1 & 7 & 15 \\
\hline Macrostemum sp. 1 & 0 & 0 & 0 & 0 & 0 & 0 & 0 & 0 & 0 & 0 & 0 & 11 & 11 \\
\hline Macrostemum sp. 2 & 0 & 0 & 73 & 0 & 0 & 0 & 0 & 7 & 0 & 0 & 0 & 4 & 84 \\
\hline Macrostemum sp. 3 & 0 & 0 & 0 & 0 & 0 & 0 & 0 & 0 & 0 & 0 & 0 & 1 & 1 \\
\hline Marilia sp. 1 & 0 & 0 & 0 & 0 & 0 & 0 & 0 & 0 & 0 & 0 & 0 & 8 & 8 \\
\hline Marilia sp. 2 & 0 & 0 & 0 & 0 & 0 & 0 & 0 & 0 & 1 & 1 & 1 & 0 & 3 \\
\hline Nectopsyche sp. 1 & 1 & 0 & 0 & 0 & 0 & 0 & 0 & 0 & 0 & 0 & 0 & 0 & 1 \\
\hline Nectopsyche sp. 2 & 0 & 0 & 0 & 0 & 0 & 2 & 1 & 0 & 3 & 3 & 0 & 0 & 9 \\
\hline Nectopsyche sp. 3 & 0 & 0 & 0 & 0 & 0 & 1 & 0 & 0 & 0 & 0 & 0 & 2 & 3 \\
\hline Nectopsyche sp. 4 & 0 & 0 & 1 & 0 & 0 & 0 & 1 & 0 & 0 & 0 & 0 & 0 & 2 \\
\hline Nectopsyche sp. 5 & 1 & 0 & 0 & 0 & 0 & 0 & 0 & 0 & 0 & 0 & 0 & 0 & 1 \\
\hline Nectopsyche sp. 6 & 0 & 0 & 0 & 0 & 0 & 0 & 0 & 0 & 0 & 1 & 0 & 0 & 1 \\
\hline Oecetis sp. 1 & 1 & 0 & 1 & 0 & 1 & 2 & 0 & 0 & 0 & 0 & 1 & 1 & 7 \\
\hline Oecetis sp. 2 & 1 & 2 & 0 & 2 & 2 & 2 & 0 & 0 & 1 & 1 & 1 & 1 & 13 \\
\hline Oecetis sp. 3 & 0 & 3 & 10 & 0 & 0 & 0 & 0 & 5 & 0 & 2 & 0 & 0 & 20 \\
\hline Oecetis sp. 4 & 2 & 0 & 1 & 0 & 2 & 0 & 2 & 1 & 0 & 5 & 0 & 0 & 13 \\
\hline Oecetis sp. 5 & 0 & 0 & 18 & 0 & 1 & 0 & 0 & 0 & 0 & 0 & 0 & 0 & 19 \\
\hline Oecetis sp. 6 & 0 & 0 & 0 & 0 & 0 & 1 & 1 & 0 & 0 & 0 & 0 & 0 & 2 \\
\hline Oxyethira sp. 1 & 0 & 0 & 1 & 0 & 0 & 0 & 0 & 0 & 0 & 0 & 0 & 0 & 1 \\
\hline Phylloicus sp. 1 & 0 & 0 & 0 & 0 & 0 & 0 & 0 & 0 & 0 & 0 & 0 & 1 & 1 \\
\hline Polyplectropus sp. 1 & 0 & 0 & 0 & 0 & 0 & 0 & 0 & 0 & 0 & 0 & 0 & 12 & 12 \\
\hline Polyplectropus sp. 2 & 0 & 0 & 0 & 0 & 0 & 0 & 0 & 0 & 1 & 0 & 0 & 0 & 1 \\
\hline Polyplectropus sp. 3 & 0 & 0 & 0 & 0 & 0 & 0 & 0 & 0 & 0 & 0 & 2 & 0 & 2 \\
\hline Smicridea (R.) sp. 1 & 0 & 0 & 1 & 0 & 0 & 0 & 0 & 0 & 0 & 0 & 0 & 6 & 7 \\
\hline Smicridea $(R$.$) sp. 2$ & 0 & 0 & 1 & 0 & 0 & 0 & 0 & 0 & 0 & 0 & 0 & 0 & 1 \\
\hline Smicridea (S.)sp. 1 & 0 & 0 & 0 & 0 & 0 & 0 & 1 & 2 & 0 & 0 & 0 & 12 & 15 \\
\hline Smicridea (S.) sp. 2 & 0 & 0 & 4 & 0 & 5 & 0 & 0 & 0 & 0 & 0 & 0 & 0 & 9 \\
\hline Smicridea (S.) sp. 3 & 0 & 0 & 1 & 0 & 0 & 0 & 0 & 0 & 0 & 0 & 0 & 0 & 1 \\
\hline Smicridea $(S$.$) sp. 4$ & 0 & 0 & 0 & 0 & 0 & 0 & 0 & 0 & 0 & 0 & 0 & 2 & 2 \\
\hline S. (S.) truncata Flint, 1974 & 13 & 0 & 0 & 0 & 0 & 0 & 1 & 0 & 0 & 1 & 0 & 1 & 16 \\
\hline Triplectides sp. 1 & 0 & 0 & 0 & 0 & 0 & 2 & 0 & 0 & 0 & 0 & 2 & 0 & 4 \\
\hline Wormaldia sp. 1 & 0 & 0 & 0 & 0 & 0 & 0 & 0 & 0 & 0 & 0 & 0 & 1 & 1 \\
\hline Total de indivíduos & 110 & 13 & 370 & 22 & 13 & 34 & 35 & 31 & 32 & 39 & 22 & 146 & 867 \\
\hline Total de Taxa & 16 & 3 & 15 & 8 & 7 & 10 & 12 & 8 & 10 & 12 & 11 & 21 & 49 \\
\hline
\end{tabular}




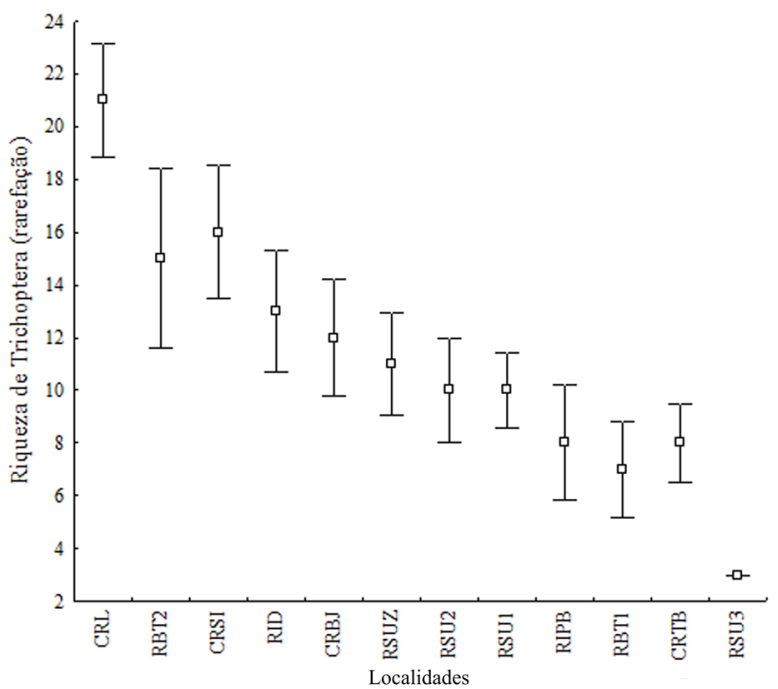

Fig. 1. Variação na riqueza estimada de espécies/morfoespécies de Trichoptera na bacia do rio Suiá-Miçú, Mato Grosso, Brasil, em 2007/2008 (CRSRI, córrego Sucuri; CRBJ, córrego Brejão; CRTB, córrego transição-brejo; CRL, córrego Lúcio; RISU1, rio Suiá-Miçú, ponto 1; RISU2, rio Suiá-Miçú, ponto 2; RISU3, rio Suiá-Miçú, ponto 3; RISUZ, rio Suiazinho; RID, rio Darro; RIPB, rio Piabanha; RIBET1, rio Betis, ponto 1; RIBET2, rio Betis, ponto 2). As barras representam um intervalo de confiança de $95 \%$.

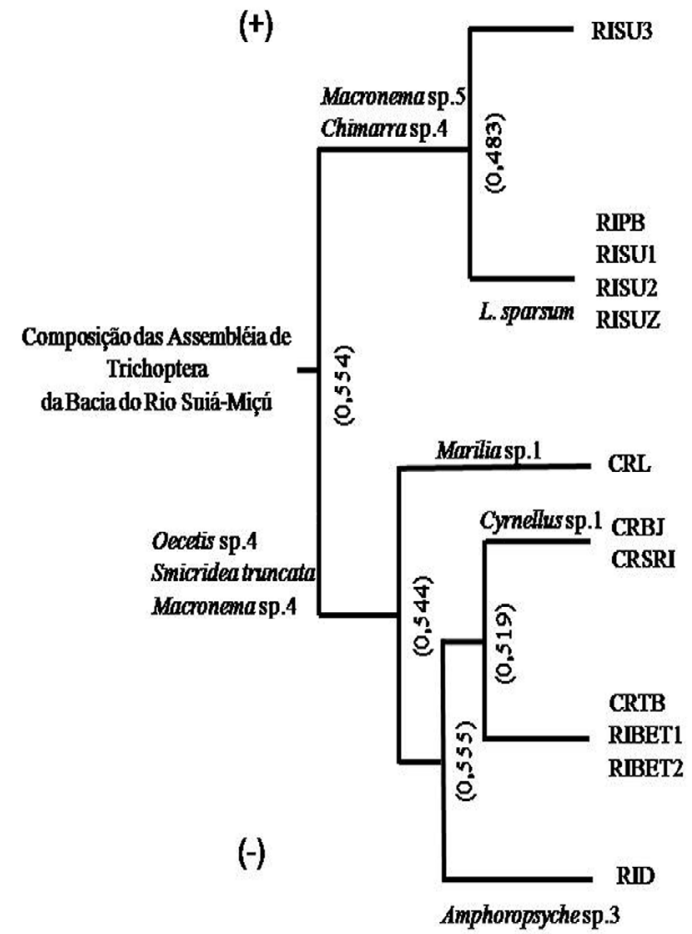

Fig. 2. Diagrama da classificação divisiva (TWINSPAN) da comunidade de larvas de Trichoptera (Insecta) amostrada em 12 locais da bacia do rio Suiá-Miçú, Mato Grosso, em 2007/2008. Nível de corte das pseudoespécies $(0,2,5,10$ e 20). Em cada divisão são apresentadas as espécies indicadoras do agrupamento formado, e os autovalores são apresentados entre parênteses (CRSRI, córrego Sucuri; CRBJ, córrego Brejão; CRTB, córrego transição-brejo; CRL, córrego Lúcio; RISU1, rio Suiá-Miçú, ponto 1; RISU2, rio Suiá-Miçú, ponto 2; RISU3, rio Suiá-Miçú, ponto 3; RISUZ, rio Suiazinho; RID, rio Darro; RIPB, rio Piabanha; RIBET1, rio Betis, ponto 1; RIBET2, rio Betis, ponto 2). temperatura, nutrientes, turbidez) (VANNOTE et al., 1980; Merrit \& Cummins, 1984). Adicionalmente, os impactos antrópicos podem refletir em modificação do padrão de abundância, perda da funcionalidade ecossistêmica, perda da diversidade biológica, desestabilização das teias tróficas e eutrofização (Allan et al., 1997; TownSEND et al., 1997).

A maior abundância de Leptoceridae e Hydropsychidae, que representaram mais de $89 \%$ da abundância na bacia do rio Suiá-Miçú, corrobora o padrão verificado em diferentes estudos no Brasil (Oliveira \& Froehlich, 1997; Oliveira \& Bispo, 2001; Bispo et al., 2006). A dominância destes grupos na escala local pode estar relacionada à sua elevada abundância e diversidade em rios da Região Neotropical (FuINT et al., 1999). Além disso, as formas imaturas de ambas as famílias habitam os mais variados tipos de ambientes aquáticos (Wiggins, 1996), o que explica sua ampla distribuição, maior abundância e riqueza nos locais estudados.

A variação da riqueza de espécies entre os locais da bacia não pode ser explicada por um único fator, podendo ser mais bem entendida quando analisada localmente. Embora a abundância do CRL tenha sido quase a metade da encontrada no RIBET2, foi mais bem distribuída entre os taxons, o que possibilitou a existência de um maior número de espécies. Este efeito pode estar relacionado à maior entrada de matéria alóctone, maior integridade e heterogeneidade de habitats provida pela vegetação ripária (BOJSEN \& JACOBSEN, 2003). No CRSRI, o segundo em riqueza, a existência de macrófitas no trecho superior e de vegetação arbórea no trecho inferior, resultou em uma maior heterogeneidade, o que pode ter possibilitado a coexistência de espécies com diferentes exigências por habitats, e assim, uma maior riqueza. No RIBET2, a elevada riqueza de espécies - assim como a maior abundância - pode estar associada à maior produtividade primária na represa logo acima do local de amostragem. Nos demais ambientes lóticos foi verificado um número intermediário de espécies (entre sete e 11 espécies). Nos ambientes brejosos um fator importante para a riqueza pode ter sido a maior produtividade e a existência de macrófitas aquáticas. No RISU3 a maior profundidade e nos ambientes impactados a elevada quantidade de sedimento fino podem explicar a baixa riqueza.

A espécie mais abundante e de ampla distribuição na bacia, L. sparsum, é filtradora generalista e bem distribuída no Brasil e na América do Sul (FLint et al., 1987; Flint et al., 1999). Esta espécie tem sido encontrada em represas próximas a escoadouros de esgoto de rios de Manaus (A.M.O. Pes, com. pess.). Sua maior tolerância aos impactos ambientais e à poluição pode explicar a alta abundância e ampla distribuição na bacia estudada. Larvas de Smicridea truncata têm sido encontradas em ambientes impactados, como rios represados e de águas ácidas e quentes ( $\left.\mathrm{pH} 4,9-5,9 ; \mathrm{T}^{\circ} 24-31^{\circ} \mathrm{C}\right)$ na região Amazônica (Pes et al., 2008), o que explica sua maior abundância no CRSRI. Já os demais membros de Hydropsychidae foram 
Tab. II. Resultado da análise de permutação da matriz de dissimilaridade de larvas de Trichoptera com relação aos preditores e suas interações (* representa a significância do teste a um nível de 0,05 ; ** Variáveis não incluídas no modelo geral da análise PER-MANOVA).

\begin{tabular}{lcccccc}
\hline & Gl & Soma de quadrados & Quadrado Médio & Razões $-F$ & $\mathrm{R}^{2}$ & $\operatorname{Pr}(>\mathrm{F})$ \\
\hline Fluxo & 1 & 0,04 & 0,04 & 2,81 & 0,14 & $0,02^{*}$ \\
Vegetação & 3 & 0,09 & 0,04 & 1,98 & 0,27 & $0,01^{*}$ \\
Fluxo: Conservação & 1 & 0,06 & 0,06 & 3,89 & 0,20 & $0,01^{*}$ \\
Conservação & 2 & 0,07 & 0,03 & 2,14 & 0,20 & 0,13 \\
IIH $^{* *}$ & 1 & 0,03 & 0,03 & 1,12 & 0,10 & 0,35 \\
Largura** & 1 & 0,03 & 0,03 & 1,16 & 0,10 & 0,30 \\
Resíduo & 2 & 0,06 & 0,02 & & 0,20 & \\
Total & 11 & 0,30 & & & 1 &
\end{tabular}

mais comuns em ambientes lóticos. Cyrnellus, que ocorreu preferencial de ambientes brejosos (CRSRI e CRBJ), apresentou distribuição distinta dos demais membros de Polycentropodidae, Polyplectropus e Cernotina, que foram registrados principalmente em ambientes lóticos. Os integrantes de Philopotamidae, Calamoceratidae e Hydroptilidae, ocorreram em baixa densidade e tiveram distribuição relacionada a córregos pequenos.

A preferência da maioria das espécies por ambientes lóticos pode estar relacionada a limitações fisiológicas, como por exemplo, baixa oxigenação e menores temperaturas da água, e a forma de aquisição de recursos alimentares (WigGins, 1996), uma vez que representam organismos filtradores. Amazonatolica hamadae Holzenthal \& Pes, 2004 é registrada para várias localidades nos estados do Amazonas, Pará, Rondônia e Bahia, principalmente em rios de águas escuras e ácidas, em áreas de floresta de terra firme não impactada (Holzenthal \& Pes, 2004). Neste estudo, $A$. hamadae foi encontrada somente no CRL, um tributário conservado e de águas claras, e constituiu o primeiro registro fora das áreas onde foi inicialmente citada desde sua descrição (Holzenthal \& Pes, 2004; Nogueira \& CABette, 2011).

Houve variação significativa na dissimilaridade de Trichoptera em função do nível de conservação, tipo de fluxo e da interação entre o fluxo e o IIH. A variação na composição faunística de tricópteros entre ambientes lóticos e lênticos e a tendência à separação relacionada ao tipo de vegetação ripária foi corroborada em ambas as análises da composição de espécies. A separação entre pequenos córregos e ambientes alagados pode ser atribuída à ausência ou mesmo menor abundância de alguns taxons em ambientes brejosos, como o CRSI, RID e CRBJ. As restrições de alguns grupos a ambientes lênticos podem estar relacionadas ao fato de muitas larvas de tricópteros serem reotáxicas (FLINT et al., 1999), ou seja, apresentam preferência por áreas de forte correnteza, como é o caso dos integrantes de Philopotamidae, Polyplectropus e Cernotina (Polycentropodidae), e a maior parte dos Hydropsychidae (WigGins, 1996; FuINT et al., 1999). Adicionalmente, alterações na composição de espécies têm sido observadas recentemente com indivíduos de Ephemeroptera nesta mesma bacia, sendo o tipo de fluxo e a conservação dos ambientes os principais fatores relacionados a modificação na riqueza e composição de espécies (Shimano et al., 2010).

As comunidades aquáticas são resistentes e resilientes frente a distúrbios previsíveis, tais como chuvas torrenciais (BRADT et al., 1999). Contudo, estas comunidades são vulneráveis a alterações abruptas do hábitat aquático quando provocadas pelas atividades humanas (TownSEND et al., 1997; BARBosa et al., 2001). Por exemplo, a retirada de vegetação favorece o aumento da sedimentação e decréscimo na entrada de matéria orgânica (Delong \& Brusven, 1994; Young \& Huryn, 1999), e tem sido relacionada à diminuição da diversidade de insetos aquáticos em diferentes estudos (TownSEND et al., 1997; Bispo \& Oliveira, 2007; Nessimian et al., 2008). Estas modificações influenciam não somente a diversidade como na estrutura da comunidade de insetos aquáticos por redução da disponibilidade de habitats ou por torná-los inadequados para a sobrevivência (Voelz \& McArThuR, 2000). Como neste estudo ao avaliarem áreas com diferentes coberturas vegetais em riachos da Amazônia, Nessimian et al. (2008) verificaram que a riqueza de EPT (Ephemeroptera, Trichoptera e Plecoptera) em áreas de pastagem foi inferior a de florestas primárias, secundárias e de fragmentos.

Foi possível constatar que as comunidades de tricópteros variaram em composição, riqueza e abundância específica devido à variabilidade das condições ambientais nos locais estudados. O tipo de fluxo, de vegetação e a conservação dos ambientes foram fatores determinantes da composição de espécies. Espécies com distribuição restrita (e.g. A. hamadae, Marilia spp.) foram amostradas principalmente em ambientes mais conservados como o CRL, e declinaram em abundância ou desapareceram em ambientes com algum tipo de alteração severa. Por outro lado, espécies tolerantes (L. sparsum, Macrostemum sp. 2, Cernotina sp. 1, Macronema sp. 4) não foram excluídas de ambientes alterados e tiveram ampla distribuição na bacia. Foi possível detectar táxons estreitamente relacionados a ambientes lênticos e lóticos ao longo da bacia. Estes resultados revelam diferentes repostas das 
espécies com relação às características dos ambientes e podem nortear o delineamento para maiores avanços nos estudos ecológicos dos insetos aquáticos da região, principalmente com vistas ao biomonitoramento da qualidade da água da bacia do rio Suiá-Miçú.

Agradecimentos. A Capes pelas bolsas concedidas à LJ e à DSN. A equipe do Laboratório de Entomologia de Nova Xavantina/ UNEMAT e ao Biólogo Herson de Souza Lima pelo suporte nas etapas de campo. Ao CNPq pelo fomento (proc. $n^{\circ}$ 520268/2005-9). Aos parceiros EMBRAPA, ISA e ONGs relacionadas à Campanha Y Ikatu Xingu, motivadora deste projeto. A Ana Maria O. Pes pelo auxílio nas identificações na revisão do texto e a dois revisores anônimos, pelas críticas e sugestões ao trabalho.

\section{REFERÊNCIAS BIBLIOGRÁFICAS}

Allan, J. D.; Erickson, D. L. \& Fay, J. 1997. The influence of catchment land use on stream integrity across multiple spatial scales. Freshwater Biology 37:149-161.

ANDERSON, M. J. 2001. A new method for non-parametric multivariate analysis of variance. Austral Ecology 26:32-46.

Angrisano, E. B. \& Korob, P. G. 2001. Trichoptera. In: Fernández, H. R. \& Domínguez, E. eds. Guia para la identificación de los artrópodos bentónicos sudamericanos. Tucumán, Editorial Universitaria de Tucumán. p. 55-92.

Barbosa, F. A. R.; Callisto, M. \& Galdean, N. 2001. The diversity of benthic macroinvertebrates as an indicator of water quality and ecosystem health: a case study for Brazil. Aquatic Ecosystem Health and Management 4:51-59.

Bispo, P. C. \& Oliveira, L. G. 2007. Diversity and structure of Ephemeroptera, Plecoptera and Trichoptera (Insecta) assemblages from riffles in mountain streams of Central Brazil. Revista Brasileira de Zoologia 24(2):283-293.

Bispo, P. C.; Oliveira, L. G.; Bini, L. M. \& Sousa, K. G. 2006. Ephemeroptera, Plecoptera and Trichoptera assemblages from riffles in mountain streams of central Brazil: environmental factors influencing the distribution and abundance of immatures. Brazilian Journal of Biology 66:611-622.

Bojsen, B. H. \& JACOBSEN, D. 2003. Effects of deforestation on macroinvertebrate diversity and assemblage structure in Ecuadorian Amazonian. Archiv für Hydrobiologie 158:317-342.

Bonada, N.; Prat, N.; Resh, V. H. \& Statzner, B. 2006. Developments in aquatic insect biomonitoring: A comparative analysis of recent approaches. Annual Review of Entomology 51:495-523.

Bradt, P.; Urban, M.; Goodman, N.; Bissell, S. \& Spiegel, I. 1999. Stability and resilience in benthic macroinvertebrate assemblages: impact of physical disturbance over twenty-five years. Hydrobiologia 403:123-133.

Brose, U.; Martinez, N. D. \& Williams, R. J. 2003. Estimating species richness: sensitivity to sample coverage and insensitivity to spatial patterns. Ecology 84(9):2364-2377.

Colwell, R. K. 2005. Estatistical estimation of species richness and shared species from samples. Version 7.5. Persistente. Disponível em: < purl.oclc..org/estimates>. Acesso em: 01.2010.

Cummins, K. W. \& Klug, M. J. 1979. Feeding ecology of stream invertebrates. Annual Review of Ecology and Systematics 10:147-172.

Cummins, K. W. \& Merritt, R. W. 2008. Ecology and Distribution of Aquatic Insects. In: Merritt, R. W. \& Cummins, K. W. eds. An introduction to the Aquatic Insects of North America. Dubuque, Kendall/Hunt Publishing Company. p. 59-65.

Delong, M. D. \& Brusven, M. A. 1994. Allochthonous input of organic matter from different riparian habitats of an agriculturally impacted stream. Environmental Management 18(1):59-71.

Dias-Silva, K.; Cabette, H. S. R.; Juen, L. \& De Marco, P. Jr. 2010. The influence of habitat integrity and physical-chemical water variables on the structure of aquatic and semi-aquatic Heteroptera. Zoologia 27(6):918-930.

Ferreira-Peruquetti, P. \& De Marco, P. JR. 2002. Efeito da alteração ambiental sobre comunidades de Odonata em riachos de Mata
Atlântica de Minas Gerais, Brasil. Revista Brasileira de Zoologia 19(2):317-327.

Flint, O. S. JR. 1974. Studies of Neotropical Caddisflies, XV: The Trichoptera of Surinam. Studies on the Fauna of Suriname and other Guyanas 14:1-151.

Flint, O. S. JR.; McAlpine, J. F. \& Ross, H. H. 1987. A revision of the Genus Leptonema Guérin (Trichoptera): Hydropsychidae: Macronematinae). Smithsonian Institution Press 450:1-193.

Flint, O. S. JR.; Rosenthal, R. W. \& Harris, S. C. 1999. Catalog of the Neotropical Caddisflies (Insecta: Trichoptera). Ohio, Ohio Biological Survey. 239p.

Heltshe, J. F. \& Forrester, N. E. 1983. Estimating species richness using the jackknife procedure. Biometrics 39:1-11.

Holzenthal, R. W. 1988. Systematics of Neotropical Triplectides (Trichoptera: Leptoceridae). Annals of the Entomological Society of America 81(2):186-208.

Holzenthal, R. W. 1995. The caddisfly genus Nectopsyche: new gemma group species from Costa Rica and Neotropics (Trichoptera: Leptoceridae). Journal of North American Benthological Society 14(1):61-83.

Holzenthal, R. W. \& Pes, A. M. O. 2004. A new genus of long-horned caddisfly from the Amazon basin (Trichoptera: Leptoceridae: Grumichellini). Zootaxa 621:1-16.

Holzenthal, R. W.; Blahnik, R. J.; Prather, A. L. \& KJer, K. M. 2007. Order Trichoptera Kirby, 1813 (Insecta), Caddisflies. Zootaxa 1668:639-698.

Hortal, J.; Borges, P. A. V. \& Gaspar, C. 2006. Evaluating the performance of species richness estimators: sensitivity to sample grain size. Journal of Animal Ecology 75:274-287.

Juen, L.; Cabette, H. S. R. \& De Marco, P. Jr. 2007. Odonate assemblage structure in relation to basin and aquatic habitat structre in Pantanal wetlands. Hydrobiologia 579:125-134.

KöPPEN, W. 1931. Grundriss der Klimakunde. Berlin, Gruyter. 338p.

MACKAY, R. J. \& Wiggins, G. B. 1979. Ecological diversity in Trichoptera. Annual Review of Entomology 24:185-208.

Maltchik, L.; Sternet, C.; Spies, M. R. \& Seigloch, A. E. 2009. Diversity and Distribution of Ephemeroptera and Trichoptera in Southern Brazil Wetlands. Journal of the Kansas Entomological Society 82(2): 160-173

McCune, B. \& Mefrord, M. J. 1999. Multivariate Analysis on the PC-ORD system. Version 5.0.1. Gleneden Beach, MjM Software.

Merritt, R. W. \& Cummins, K. W. 1984. An Introduction to the aquatic insects of North America. Dubuque, Kendall / Hunt Publishing Company. 721p.

MiLne, M. 1938. The "metamorphotype method" in Trichoptera. Journal of the New York Entomological Society 46:435-437.

Morton, D. C.; DeFries, R. S.; Shimabukuro, Y. E.; Anderson, L. O.; Arai, E.; Espirito-Santo, F. B.; Freitas, R. \& Morisette, J. 2006. Cropland expansion changes deforestation dynamics in the southern Brazilian Amazon. PNAS 103(39):14637-14641.

Nessimian, J. L.; Venticinque, E. M.; Zuanon, J.; De Marco, P. Jr.; Gordo, M.; Fidelis, L.; Batista, J. D. \& Juen, L. 2008. Land use, habitat integrity, and aquatic insect assemblages in Central Amazonian streams. Hydrobiologia 614(1):117-131.

Nogueira, D. S. \& CABETte, H. S. R. 2011. New records and notes on geographic distribution of Trichoptera Kirby, 1813 (Insecta) from the State of Mato Grosso, Brazil. Biota Neotropica 11(2).

Oksanen, J.; Blanchet, F. G.; Kindt, R.; Legendre, P.; O’Hara, R. B.; Simpson, G. L.; Solymos, P.; Stevens, M. H. H. \& Wagner, H. 2010. Vegan: community ecology package. $R$ package version $\mathbf{1 . 1 7 - 3}$. Disponível em: $<$ http://cran.r-project.org $>$. Acesso em: 23.06.2010.

OliveirA, L. G. \& Bispo, P. C. 2001. Ecologia de larvas de Trichoptera Kirby (Insecta) em dois córregos de primeira ordem da Serra dos Pirineus, Pirinópolis, Goiás, Brasil. Revista Brasileira de Zoologia 18(4): 1245-1252.

Oliveira, L. G. \& Froehlich, C. G. 1997. Diversity and community structure of aquatic insects (Ephemeroptera, Plecoptera and Trichoptera) in a mountain stream in a Southeastern Brazil. Acta Limnologica Brasiliensis 9:139-148. 
Paprocki, H.; Holzenthal, R. W. \& BlahniK, R. J. 2004. Checklist of the Trichoptera (Insecta) of Brazil I. Biota Neotropica 4(1):1-22.

Pes, A. M. O.; Hamada, N. \& Nessimian, J. L. 2005. Chaves de identificação de larvas para famílias e gêneros de Trichoptera (Insecta) da Amazônia Central, Brasil. Revista Brasileira de Entomologia 49(2): 181-204.

Pes, A. M. O.; Hamada, N. \& Soares, C. C. 2008. Description of the last-instar larva and pupa and the bionomics of Smicridea (Smicridea) truncata Flint (Trichoptera: Hydropsychidae) in Central Amazonia, Brazil. Zootaxa 1732:56-60.

Pongratz, J.; Bounoua, L.; DeFries, R. S.; Morton, D. C.; Anderson, L. O.; MAuser, W. \& KlinK, C. A. 2006. The impact of land cover change on surface energy and water balance in Mato Grosso, Brazil. Earth Interactions 10:1-17.

R Development Core Team. 2010. R: a language and environment for statistical computing. R Foundation for Statistical Computing, Vienna, Austria. Disponível em: <http://www.rproject.org $>$. Acesso em: 23.06.2010.

Ratter, J. A.; Askew, G. P.; Montgomery, R. F. \& Gifford, D. R. 1978 Observations on the vegetation of northeastern Mato Grosso II. Forest and Soils of the Rio Suiá-Miçú area. Proceedings of the Royal Society of London 203(B):191-208.

Rosenberg, D. M. \& Resh, V. H. 1993. Introduction to freshwater biomonitoring and benthic macroinvertebrates. In: ROSENBERG, D. M. \& Resh, V. H. eds. Freshwater biomonitoring and benthic macroinvertebrates. New York, Chapman \& Hall. p. 1-9.

Santos, A. P. M.; Dumas, L. L.; Jardim, G. A. \& Nessimian, J. L. 2011. Brazilian Caddisflies: Checklists and Bibliography. Disponível em: <https://sites.google.com/site/braziliancaddisflies $>$. Acesso em: 27.06.2011.

Shimano, Y.; Cabette, H. S. R.; Salles, F. F. \& Juen, L. 2010 Composição e distribuição da fauna de Ephemeroptera (Insecta) em área de transição Cerrado-Amazônia, Brasil. Iheringia, Série Zoologia 100(4):301-308.

Strahler, H. N. 1957. Quantitative analysis of watershed geomorphology. American Geophysical Union Transactions 38:913-920.

Townsend, C. R.; Arbuckle, C. J.; Crowl, T. A. \& Scarsbrook, M. R. 1997. The relationship between land use and physicochemistry, food resources and macroinvertebrate communities in tributaries of the Taieri River, New Zealand: a hierarchically scaled approach. Freshwater Biology 37:177-191.

Vannote, R. L.; Minshall, G. W.; Cummins, K. W.; Sedell, J. R.; \& Cushing, C. E. 1980. The River Continuum Concept. Canadian Journal of Fisheries and Aquatic Sciences 37:130-137.

Voelz, N. J. \& McArthur, J. V. 2000. An exploration of factors influencing lotic insect species richness. Biodiversity and Conservation 9:1543-1570.

Wantzen, K. M.; Siqueira, A.; Nunes Da Cunha, C. \& SÁ, M. F. P. 2006. Stream-valley systems of the Brazilian Cerrado: impact assessment and conservation scheme. Aquatic Conservation: Marine and Freshwater Ecosystems 16:713-732.

WigGINS, G. B. 1996. Larvae of the North American Caddisfly Genera (Trichoptera). Toronto, Univesity of Toronto Press. $457 \mathrm{p}$.

Young, R. G. \& Huryn, A. D. 1999. Effects of land use on stream 